Pacific Journal of Mathematics

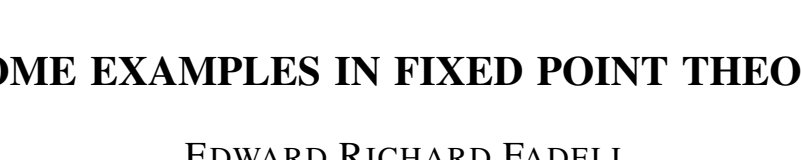




\title{
SOME EXAMPLES IN FIXED POINT THEORY
}

\author{
E. FADELL
}

It is known that the fixed point property (f.p.p.) is not invariant under suspension and join in the category of simply connected polyhedra. In this paper we exhibit examples to show that f.p.p. is not invariant under suspension and join in the category of simply connected polyhedra satisfying the Shi condition and more strongly, in the category of simply connected compact manifolds. We also exhibit a simply connected polyhedron $X$ such that the smash product $X \wedge X$ fails to have f.p.p. if one choice of base point is used to form $X \wedge X$, while $X \wedge X$ has f.p.p. using another choice of base point. In the last section we prove that f.p.p. is invariant under Cartesian products in very special circumstances.

It is known that the fixed point property (f.p.p.) in the category of simply connected polyhedra is not an invariant under cartesian products, smash products, suspension, join or homotopy type (Lopez [3] and [1]). In all cases the counterexamples are based upon polyhedra which fail to satisfy the Shi condition, namely that for each vertex $v, \partial S t v$ (boundary of the star of $v$ ) be connected and the dimension is $\geqq 3$. It is therefore natural to consider the behavior of f.p.p. in more restrictive categories. As suggested in [1], one should look at f.p.p. in the following categories:

$\mathscr{S}$ : Polyhedra satisfying the Shi condition.

$\mathscr{S}_{0}$ : Simply connected polyhedra in $\mathscr{S}$.

$\mathscr{C l}$ : Compact topological manifolds, dimension $\geqq 3$.

$\mathscr{C l}_{0}$ : Simply connected manifolds in $\mathscr{C}$.

In the categories $\mathscr{S}$ and $\mathscr{C l}$ f.p.p. is a homotopy type invariant. In fact, if $X$ is any compact ANR dominated by $Y$, where $Y$ is in $\mathscr{S}$ or $\mathscr{C l}$, then $Y$ f.p.p. implies $X$ f.p.p. [1]. Thus the result, $Y$ f.p.p. implies $Y \times I$ f.p.p., is valid in the categories $\mathscr{S}$ or $\mathscr{C}$ even though it is false for (simply connected) polyhedra in general.

The question

$$
X \text { f.p.p., } Y \text { f.p.p. } \Longrightarrow X \times Y \text { f.p.p.? }
$$

in the categories $\mathscr{S}$ or $\mathscr{C}$ remains open. In $\S 4$, we prove two very special cases for the categories $\mathscr{S}_{0}$ and $\mathscr{C}_{0}$. In $\S 2$ we provide the details of the examples announced in [1] which show that in $\mathscr{S}_{0}$ and $\mathscr{C}_{0}$ f.p.p. is not invariant under the suspension and join operations. In $\S 3$ we use one of the examples of $\S 2$ to construct a simply connected polyhedron $X$ which has f.p.p. and with the curious property 
that with one choice of base point $(a, a)$ the resulting smash product $X \wedge X=X \times X / a \times X \cup X \times a$ fails to have f.p.p., while constructing $X \wedge X$ with another choice of base point preserves f.p.p.

2. Two examples, If $F: X \rightarrow X$ is a self-map of a compact connected metric ANR, then for any field $\Lambda$

$$
L(f ; \Lambda)=\sum_{k}(-1)^{k} \text { Trace } f_{k}^{*}
$$

is the Lefschetz number of $f$ over $\Lambda$ and $\bar{L}(f, \Lambda)=L(f, \Lambda)-1$ is the reduced Lefschetz number of over $\Lambda$. When $\Lambda=Q$, the field of rational numbers, then $L(f)=L(f, Q)$ is the usual Lefschetz number of $f$. $\chi(X)$ and $\bar{\chi}(X)=\chi(X)-1$ will denote the Euler characteristic and reduced Euler characteristic, respectively. All spaces in this paper will be connected compact metric ANR's.

We will make use of the following simple lemma.

Lemma 2.1. Suppose 1 is a field of characteristic $p \neq 2$ and $X$ and $Y$ are spaces with the property that for every self-map $f: X \rightarrow X$, $\bar{L}(f ; \Lambda)=0$ or 1 and every self-map $g: Y \rightarrow Y, \bar{L}(g, \Lambda)=0$. Then any space $W \sim X \vee Y$ has f.p.p.

Proof. Let

$$
\begin{aligned}
& X \stackrel{i_{1}}{\longrightarrow} X \vee Y \stackrel{r_{1}}{\longrightarrow} X \\
& Y \stackrel{i_{2}}{\longrightarrow} X \vee Y \stackrel{r_{2}}{\longrightarrow} Y
\end{aligned}
$$

denote the natural inclusions and retractions. Then, if $\varphi: X \vee Y \rightarrow$ $X \vee Y$ is any map, let $f=r_{1} \varphi i_{1}$ and $g=r_{2} \varphi i_{2}$. It is easy to verify that

$$
\bar{L}(\varphi, \Lambda)=\bar{L}(f, \Lambda)+\bar{L}(g, \Lambda)=0 \text { or } 1 .
$$

Therefore, $L(\varphi, \Lambda) \neq 0$. Thus, $X \vee Y$ has the property that every self-map $\varphi$ has nonzero Lefschetz number over $\Lambda$. Since this property is a homotopy type invariant, it follows that if $W \sim X \vee Y$, then $W$ has f.p.p.

LEMma 2.2. If $H P^{4}$ is quaternionic projective 4-space, then for every self-map $f: H P^{4} \rightarrow H P^{4}, \bar{L}\left(f, Z_{3}\right)=0$ or 1 .

Proof. Let $u$ denote a generator in $H^{4}\left(H P^{4} ; Z_{3}\right)$. Then, if $f^{*}(u)=a u$,

$$
\bar{L}\left(f ; Z_{3}\right)=a+a^{2}+a^{3}+a^{4}=0 \text { or } 1 .
$$


LEMma 2.3. If $S H P^{3}$ is the suspension of quaternionic projective 3-space, then for every self-map $g: S H P^{3} \rightarrow S H P^{3}, \bar{L}\left(g ; Z_{3}\right)=0$.

Proof. Choose a generator $v \in H^{5}\left(S H P^{3} ; Z^{3}\right)$ such that $P^{1} v$ and $P^{2} v$ generate the $Z_{3}$-cohomology in dimensions 9 and 13 , respectively. $P^{i}$ is the mod 3 Steenrod reduced power operator. Now, if $g: S H P^{3} \rightarrow$ $S H P^{3}$ and $g^{*}(v)=b v$,

$$
\bar{L}\left(g ; Z_{3}\right)=b+b+b=0 .
$$

Proposition 2.4. Any space $W \sim H P^{4} \vee S H P^{3}$ has f.p.p.

Proposition 2.5. Let

$$
K=H P^{4} \cup_{I} S H P^{3}
$$

denote the union of $H P^{4}$ and $S H P^{3}$ along an edge. Then, $K$ is a simply connected polyhedron which has f.p.p. and satisfies the Shi condition. Moreover, $\chi(K)=2$.

REMARK. $\quad K^{\prime}=\left(H P^{4} \vee S H P^{3}\right) \times I$ has the same properties as $K$.

Proposition 2.6. The suspension $S K$ and the join $K \circ K$ fail to have f.p.p.

Proof. Since $\bar{\chi}(S K)=-\bar{\chi}(K)$ and $\bar{\chi}(K \circ K)=-\bar{\chi}(K) \bar{\chi}(K)$, both $S K$ and $K \circ K$ have Euler characteristic 0 . Since $S K$ and $K \circ K$ satisfy the Shi condition, both admit maps homotopic to the identity map which are fixed point free [5].

THEOREM 2.7. The f.p.p. is not invariant under suspension and join in the category $\mathscr{S}_{0}$.

Our next example will verify the above theorem in the category $\mathscr{C l}_{0}$.

Let $q: S^{7} \rightarrow S^{4}$ denote the standard Hopf fibering and let $A=M_{1}(q)$, $B=M_{2}(q)$ denote two copies of the mapping cylinder of $q$. Then if $h: S^{7} \rightarrow S^{7}$ is a reflection (degree -1 ), where $S^{7}$ is identified with one end of the mapping cylinder of $q$, we may represent the connected sum

$$
M=H P^{2} \# H P^{2}
$$

by

$$
M=A \cup_{h} B
$$


There is a natural "flip" map $f: M \rightarrow M$ which takes $A$ to $B$ and $B$ to $A$ and which is the reflection on $S^{7}=A \cap B$, where $A$ and $B$ are identified with the appropriate subsets of $M$. It is easy to see that $f$ is a homeomorphism which preserves orientation. Furthermore, by identifying $S^{7}=A \cap B$ we obtain an identification map

$$
g: M \longrightarrow H P^{2} \vee H P^{2}
$$

which allows us to compute the cohomology ring structure ( $Z$-coefficients) as follows:

Lemma 2.8. The cohomology of $M=H P^{2} \# H P^{2}$ is given by

$$
\begin{aligned}
& H^{0}(M)=Z, \text { generator } 1 \\
& H^{2}(M)=Z \oplus Z, \text { generators } x, y \\
& H^{4}(M)=Z, \text { generator } x^{2}=y^{2}
\end{aligned}
$$

with $H^{q}(M)=0$ in the remaining dimensions and $x y=0$.

Theorem 2.9. $M=H P^{2} \# H P^{2}$ is a simply connected manifold with f.p.p. which admits a map $f$ of Lefschetz number $L(f)=2$.

Proof. The natural "flip" map $f: M \rightarrow M$ defined above has $L(f)=2$ so that the last part of the theorem is easy. Now, let

$$
\varphi: M \longrightarrow M
$$

denote an arbitrary map and suppose, using (10), that

$$
\begin{aligned}
& \varphi^{*}(x)=a x+b y \\
& \varphi^{*}(y)=c x+d y .
\end{aligned}
$$

Then,

$$
\varphi^{*}\left(x^{2}\right)=\varphi^{*}\left(y^{2}\right)=\left(a^{2}+b^{2}\right) x^{2}=\left(c^{2}+d^{2}\right) y^{2}
$$

and

$$
\varphi(x y)=0=(a c+b d) x^{2}
$$

which yields the conditions

$$
a^{2}+b^{2}=c^{2}+d^{2}, \quad a c+b d=0 .
$$

Furthermore,

$$
L(\varphi)=1+a+d+a^{2}+b^{2} .
$$

We now consider individual cases. 
Case 1. $a=0, b=0$. Here $L(\varphi)=1$.

Case 2. $a^{2}+b^{2} \neq 0,(a, b) \neq(-1,0)$. Using (15), we have

$$
a^{2}\left(a^{2}+b^{2}\right)=a^{2}\left(c^{2}+d^{2}\right)=d^{2}\left(a^{2}+b^{2}\right)
$$

so that $a= \pm d$. If $a=-d, L(\varphi)=1+a^{2}+b^{2}>0$. On the other hand if $a=d, L(\varphi)=(1+a)^{2}+b^{2}>0$.

Case 3. $a=-1, b=0$. This case does not occur. To see this, choose $v \in H^{4}\left(H P^{2} ; \mathbf{Z}_{3}\right)$ such that $P^{1} v=v^{2}$. Then we may assume $g^{*}(v)=x$ (over $\mathbf{Z}_{3}$ ) and $P^{1} x=x^{2}$ in $H^{4}\left(M ; \mathbf{Z}_{3}\right.$ ). If $\phi^{*}(x)=a x$ (over $\mathbf{Z}$ ), we must have

$$
\varphi^{*}\left(P^{1} x\right)=\varphi^{*}\left(x^{2}\right)=a^{2} x^{2}=a^{2} P^{1} x=a P^{1} x=a x^{2}
$$

so that $a^{2} \equiv a(\bmod 3)$. This precludes $a=-1$.

Thus, we see that for any map $\varphi: M \rightarrow M, L(\varphi) \neq 0$ and hence $M$ has f.p.p.

THEOREM 2.10. The f.p.p. is not invariant under suspension and join in the category $\mathscr{M}_{0}$.

Proof. Let $M$ denote the manifold in the previous theorem and $f: M \rightarrow M$ the map with $L(f)=2$. Then,

$$
S f: S M \longrightarrow S M \text { and } f \circ f: M \circ M \longrightarrow M \circ M
$$

yield

$$
\bar{L}(S f)=-\bar{L}(f)=-1=-\bar{L}(f) \bar{L}(f)=\bar{L}(f \circ g)
$$

so that

$$
L(S f)=0=L(f \circ f) .
$$

Since we are in the simply connected case, the Nielson number of $S f$ (and $f \circ f$ ) is zero. Therefore again using [5], $S f$ and $f \circ f$ can be deformed to fixed point free maps so that $S M$ and $M \circ M$ fail to have f.p.p.

3. The f.p.p. and smash product. Our objective in this section is to show that there is a simply connected polyhedron $X$ with f.p.p. such that the smash product $X \wedge X=X \times X / X \vee X$ has f.p.p. with one choice of base point $x_{0} \in X$ while it may fail to have f.p.p. if one employs another base point $x_{1} \in X$.

We will make use of the polyhedron 


$$
K=H P^{4} \cup_{I} S H P^{3}
$$

discussed in the previous section. If $N=S H P^{2}$ and

$$
X=K \vee N=\left(H P^{4} \cup_{I} S H P^{3}\right) \vee S H P^{2}
$$

we will show that $X \wedge X$ fails to have f.p.p. if the base point $x_{0} \in X$ is chosen distinct from the wedge point $v \in X$. On the other hand, if the wedge point $v$ is employed to form $X \wedge X$, then $X \wedge X$ retains f.p.p.

THeOREM 3.1. If $x_{0} \neq v$, then

$$
X \wedge X=X \times X / x_{0} \times X \cup X \times x_{0}
$$

fails to have f.p.p.

Proof. First we observe that since $\chi(X)=0, \bar{L}(i d)=-1$, where $\bar{L}$ is the reduced Lefschetz number. Since $\bar{\chi}(K)=1$ (reduced Euler characteristic) we see that $X$ admits a map $g$ such that $\bar{L}(g)=1$. Thus, $\bar{L}(i d \wedge g)=\bar{L}(i d) \bar{L}(g)=-1$, and we see that $f=i d \wedge g$ is a self-map of $X \wedge X$ with $L(f)=0 . \quad X \wedge X$ is simply connected and can be shown to satisfy the Shi condition (using the fact that $x_{0} \times X \cup X \times x_{0}$ fails to separate $\left.X \times X\right)$. It follows that there is a map $g \sim f$ such that $g$ has no fixed points. Thus, $X \wedge X$ fails to have f.p.p.

We now show that using the wedge point $v$

$$
X \wedge X=X \times X / v \times X \cup X \times v
$$

has f.p.p. Although the details are lengthy, the idea is quite simple. $X=K \cup N$ with $K \cap N=v$, the wedge point. Using $v$ as base point in the formation of $X \wedge X$ yields

$$
X \wedge X=(K \wedge K) \vee(K \wedge N) \vee(N \wedge K) \vee(N \wedge N)
$$

where the four-fold wedge on the right is understood to have a single wedge point $v^{\prime}$ corresponding to $v \times X \cup X \times v$. Now, since f.p.p. is invariant under the wedge operation, it suffices to show that the four individual wedge factors $K \wedge K, K \wedge N, N \wedge K, N \wedge N$ have f.p.p.

Lemma 3.2. $H P^{4} \wedge H P^{4}$ has f.p.p. Specifically, for any self map $\varphi, \bar{L}\left(\varphi, Z_{3}\right)=0$ or 1 .

Proof. We will identify $H^{*}(A \wedge B)$ with $H^{*}(A \times B, A \vee B) \simeq$ $H^{*}\left(A, a_{0}\right) \otimes H^{*}\left(B, b_{0}\right)$ using always field coefficients. Then, working over $Z_{3}, H^{*}\left(H P^{4}\right)$ has a basis of the form 
where $P^{i}$ is the Steenrod reduced power operator. Then, we may arrange a basis for $H^{*}\left(H P^{4} \wedge H P^{4}\right)$ in positive dimensions as follows:

$$
\begin{array}{rrrr}
\alpha \times \alpha & \alpha \times P^{1} \alpha+P^{1} \alpha \times \alpha & \alpha \times P^{2} \alpha+P^{1} \alpha \times P^{1} \alpha+P^{2} \alpha \times \alpha \\
P^{1} \alpha \times \alpha & P^{1} \alpha \times P^{1} \alpha-P^{2} \alpha \times \alpha & -P^{2} \alpha \times P^{1} \alpha+P^{1} \alpha \times P^{2} \alpha \\
\alpha \times P^{2} \alpha & P^{1} \alpha \times P^{2} \alpha & P^{2} \alpha \times P^{2} \alpha \\
\alpha^{4} \times \alpha & \alpha^{4} \times P^{1} \alpha & \alpha^{4} \times P^{2} \alpha \\
\alpha \times \alpha^{4} & P^{1} \alpha \times \alpha^{4} & P^{2} \alpha \times \alpha^{4} . \\
\alpha^{4} \times \alpha^{4} & & &
\end{array}
$$

Notice that (for the first five rows) applying $P^{1}$ and $P^{2}$ to the first column yields the second and third columns. This means that for a self-map $\varphi$ : of $H P^{4} \wedge H P^{4}, \bar{L}\left(\varphi, Z_{3}\right)=\lambda^{4}$, where $\varphi^{*}(\alpha \times \alpha)=\lambda(\alpha \times \alpha)$. This concludes the proof.

LemMA 3.3. $H P^{4} \wedge S H P^{3}$ has f.p.p. Specifically, for any self$\operatorname{map} \varphi, \bar{L}\left(\varphi, Z_{3}\right)=0$.

Lemma 3.4. $S H P^{3} \wedge S H P^{3}$ has f.p.p. Specifically, for any self$\operatorname{map} \rho, \bar{L}\left(\varphi, Z_{3}\right)=0$.

The proofs of these lemmas are modelled after the proof of Lemma 3.2 and consequently are left as exercises.

Proposition 3.5. $K \wedge K$ has f.p.p.

Proof. Let $K^{\prime}=H P^{4} \vee S H P^{3}$, then using the above lemmas every self-map $\varphi^{\prime}$ of

$$
\begin{aligned}
& K^{\prime} \wedge K^{\prime} \\
& \quad=\left(H P^{4} \wedge H P^{4}\right) \vee\left(H P^{4} \wedge S H P^{3}\right) \vee\left(S H P^{3} \wedge H P^{4}\right) \vee\left(S H P^{3} \wedge S H P^{3}\right)
\end{aligned}
$$

has the property that $\bar{L}\left(\varphi, Z_{3}\right)=0$ or 1 (using the technique in the proof of Lemma 2.1). Since this property is a homotopy type invariant, every self-map $\varphi$ of $K \wedge K$ has $L\left(\varphi, Z_{3}\right) \neq 0$. Thus, $K \wedge K$ has f.p.p.

Lemma 3.6. $H P^{4} \wedge S H P^{2}$ has f.p.p. Specifically, for every self$\operatorname{map} \varphi, \bar{L}\left(\varphi, Z_{2}\right)=0$.

Proof. We may choose basis for the $Z_{2}$-cohomology of $H P^{4}$ and $S H P^{2}$, respectively, as follows

$$
H P^{4}: 1, \alpha, S q^{4} \alpha, \beta, S q^{4} \beta
$$




$$
S H P^{2}: 1, u, S q^{4} u \text {. }
$$

Then, we may arrange a basis (in positive dimensions) for the $Z_{2}$ cohomology of $H P^{4} \wedge S H P^{2}$ as follows

$$
\begin{array}{rrrl}
\alpha \times u & \alpha \times S q^{4} u+S q^{4} \alpha+S q^{4} \alpha \times u \\
S q^{4} \alpha \times u & S q^{4} \alpha \times S q^{4} u \\
\beta \times u & \beta \times S q^{4} u+S q^{4} \beta \times u \\
S q^{4} \beta \times u & S q^{4} \beta \times S q^{4} u &
\end{array}
$$

where $S_{q}^{4}$ applied to the first column yields the second coiumn. This is enough to show that for every self-map $\varphi, \bar{L}\left(\varphi, Z_{2}\right)=0$.

LEMMA 3.7. $S H P^{3} \wedge S H P^{2}$ has f.p.p. Specifically, for every self$\operatorname{map} \varphi, \bar{L}\left(\varphi, Z_{2}\right)=0$.

The proof of this lemma is similar to the proof of Lemma 3.6.

Proposition 3.8. $K \wedge N$ has f.p.p.

Proof. $K \wedge N$ has the same homotopy type as

(9) $W=\left(H P^{4} \vee S H P^{3}\right) \wedge S H P^{2}=\left(H P^{4} \wedge S H P^{2}\right) \vee\left(S H P^{3} \wedge S H P^{2}\right)$.

But by the previous lemmas, every self-map $\varphi^{\prime}$ of $W$ has the property that $\bar{L}\left(\varphi^{\prime}, Z_{2}\right)=0$ and hence every self-map of $K \wedge N$ has Lefschetz number 1 (over $Z_{2}$ ). Thus, $K \wedge N$ has f.p.p.

Proposition 3.9. $N \wedge N$ has f.p.p.

Proof. Working with $Z_{2}$ coefficients, a basis for the cohomology of $N=S H P^{2}$ has the form $1, u, S q^{4} u$. A basis for the cohomology (in positive dimensions) of $N \wedge N$ can be written

$$
\begin{array}{rl}
u \times u & S q^{4} u \times u+u \times S q^{4} u \\
S q^{4} u \times u & S q^{4} u \times S q^{4} u
\end{array}
$$

where $S q^{4}$ applied to column one yields column two. This, given any self-map $\varphi$ of $M, \bar{L}\left(\varphi ; Z_{2}\right)=0$.

THEOREM 3.10. Using the wedge point $v$ of $X$

$$
X \wedge X=X \times X / v \times X \cup X \times v
$$

has f.p.p. 
4. Very special cases of the product theorem. Consider the following property:

Property $F: X$ is said to have property $F$ if, and only if, $L(f) \neq 0$ for every self-map $f: X \rightarrow X$.

In terms of this property we recall the following theorem [1]:

THEOREM 4.1. If $X$ belongs to $\mathscr{S}_{0}$ or $\mathscr{C l}_{0}$, then $X$ has f.p.p. if, and only if, $X$ has property $F$.

Thus for spaces in $\mathscr{S}_{0}$ (or $\mathscr{C}_{0}$ ), the question of the invariance of f.p.p. under Cartesian products (see (1) of $\S 1$ ) is equivalent to the question

$X$ and $Y$ have property $F \Rightarrow X \times Y$ has property $F$ ?

Our next theorem answers (1) in the affirmative under quite special hypothesis. In the following we use rational singular cohomology.

THEOREM 4.2. Suppose $X$ and $Y$ are spaces having property $F$. Suppose further that $X$ has trivial cup products and $X$ and $Y$ have disjoint cohomology, i.e., $H^{p}(X) \neq 0, H^{q}(Y) \neq 0, p, q \geqq 1$, implies $p \neq q$. Then $X \times Y$ has property $F$.

We will make use of the following lemma whose proof is left to the reader.

Lemma 4.3. Suppose $\psi: X \rightarrow Y$ is a map and $\psi_{0}: Y \rightarrow Y$ is defined by the diagram

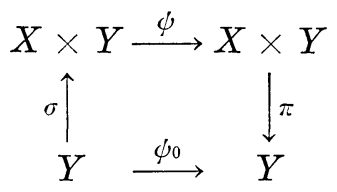

where $\sigma$ is a section given by $\sigma(y)=\left(x_{0}, y\right), x_{0} \in X$ and $\pi$ is a projection on the second factor. Then, for $v \in H^{n}(Y)$

$$
\psi^{*}(1 \times v)=1 \times \psi_{0}^{*}(v)+E(v)
$$

where $E(v)$ is a linear combination of terms of the form $a \times b$ where $\operatorname{dim} a \geqq 1$.

Proof of 4.2. Let $\varphi: X \times Y \rightarrow X \times Y$ denote an arbitrary map and let $f$ and $g$ be defined by the diagrams 


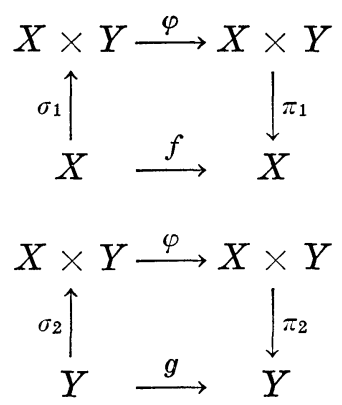

where $\sigma_{1}$ and $\sigma_{2}$ are sections and $\pi_{1}$ and $\pi_{2}$ are projections (see Lemma 4.3).

We choose bases $1=u_{1}, \cdots, u_{k}$ and $1=v_{1}, \cdots, v_{l}$ for the rational cohomology of $X$ and $Y$, respectively. Then, elements of the form $u_{i} \times v_{j}$ form a basis for the cohomology of $X \times Y$. If $u$ and $v$ are typical basis elements, then using Lemma 4.3

$$
\begin{aligned}
& \varphi^{*}(u \times 1)=f^{*}(u) \times 1+E(u) \\
& \varphi^{*}(1 \times v)=1 \times g^{*}(v)+E(v)
\end{aligned}
$$

where $E(u)$ is a linear combination of terms of the form $a \times b$ with $\operatorname{dim} b \geqq 1$ and $E(v)$ is a linear combination of terms of the form $a^{\prime} \times b^{\prime}$, $\operatorname{dim} a^{\prime} \geqq 1$. Suppose $\operatorname{dim} u=m$ and $\operatorname{dim} v=n$. Then

$$
\begin{aligned}
& \varphi^{*}(u \times v) \\
& \quad=f^{*}(u) \times g^{*}(v)+E(u)\left(1 \times g^{*}(v)\right)+\left(f^{*}(u) \times 1\right) E(v)+E(u) E(v) .
\end{aligned}
$$

Now $E(u)$ is a linear combination of terms of the form $a \times b$ where $\operatorname{dim} a \leqq m-1$ so that $u \times v$ cannot appear in the term $E(u)\left(1 \times g^{*}(v)\right)$. Similarly, $u \times v$ cannot appear in the term $\left(f^{*}(u) \times 1\right) E(v)$. In $E(u) E(v)$ a typical term has the form

$$
(a \times b)\left(a^{\prime} \times b^{\prime}\right)= \pm a a^{\prime} \times b b^{\prime}
$$

where $\operatorname{dim} a \leqq m-1, \operatorname{dim} b \geqq 1, \operatorname{dim} a^{\prime} \geqq 1, \operatorname{dim} b^{\prime} \leqq n-1$. If $\operatorname{dim} a \geqq 1$, $a a^{\prime}=0$ so that (4) is 0 . On the other hand if $\operatorname{dim} a=0$ then $\operatorname{dim} b=m$. Since $\operatorname{dim} u=m$ we see that $b=0$ and hence (4) is 0 in this case. Thus $E(u) E(v)=0$. Thus, we see that $\varphi^{*}(u \times v)$ and $(f \times g)^{*}(u \times v)$ have the same coefficient of $u \times v$. Thus,

$$
L(f \times g)=L(f) L(g)=L(\varphi) \neq 0 .
$$

Theorem 4.4. Suppose $X$ and $Y$ belong to $\mathscr{S}_{0}\left(\right.$ or $\mathscr{M}_{0}$ ) and have f.p.p. Then $X \times Y$ has f.p.p. if $X$ or $Y$ has trivial rational cup products and $X$ and $Y$ have disjoint rational cohomology. 
ExAmPLE. Using Theorem 4.4, we see that $C P^{i} \times S C P^{j}$ has f.p.p. for $i$ and $j$ even, $i, j \geqq 2$. To prove that $C P^{i}$ has f.p.p., arrange a basis for the $Z_{2}$-cohomology of $C P^{i}$ in the form ( $i$ even)

$$
1, x_{1}, S q^{2} x_{1}, x_{2}, S q^{2} x_{2}, \cdots
$$

so that for any self-map $\varphi$ of $C P^{i}$ we have $L\left(\varphi, Z_{2}\right)=1$. Since $S_{q}$ commutes with suspension the same argument works for $S C P^{i}$.

Theorem 4.4 raises the following question:

QUESTION 4.5. If $S X \times Y$ has f.p.p., does this imply that $X \times Y$ has f.p.p.?

An affirmative answer to this question would settle the following conjecture.

Conjecture 4.6. Suppose $X$ and $Y$ belong to $\mathscr{S}_{0}$ and $X$ and all its suspension have f.p.p. Then if $Y$ has f.p.p., so does $X \times Y$.

The technique used to prove Theorem 4.2 can also be used to prove the following.

THEOREM 4.7. Suppose $X$ and $Y$ belong to $\mathscr{S}_{0}\left(\right.$ or $\left.\mathscr{L l}_{0}\right)$ and have f.p.p. Suppose further that $H^{*}(X)$ is a truncated polynomial ring on a single generator $u \in H^{k}(X)$. Then, if $H^{k}(Y)=0, X \times Y$ has f.p.p.

Example. $C P^{i} \times H P^{j}$, where $i$ is even $(i, j \geqq 2)$ has f.p.p. The argument that $H P^{j}$ has f.p.p. goes as follows. First of all, if $\varphi$ is a self-map of $H P^{j}$, then working over the rational field

$$
L(\varphi)=1+a+a^{2}+\cdots+a^{j}
$$

where $\varphi^{*}(u)=a u, u$ a generator in $H^{4}\left(H P^{j}\right)$. Of course, if $j$ is even we're done, since $L(\phi) \neq 0$ in this case. If $j$ is odd, $j \geqq 3$ we need only preclude the case $a=-1$. Working over $Z_{3}$, we may assume that $P^{1} u=u^{2}$ in $H^{8}\left(H P^{j} ; Z_{3}\right)$. This forces

$$
a^{2} \equiv a(\bmod 3)
$$

which precludes $a=-1$.

REMARK. G. Bredon was the first to observe that $H P^{3}$ has f.p.p. using the above argument. 


\section{BIBLIOGRAPHY}

1. E. Fadell, Recent results in the fixed point theory of continuous maps, Invited Address, Cincinatti, April, 1969.

2. Jiang Bo-Ju, Estimation of Nielsen numbers, Chinese Math. 5 (1964), 330-339.

3. W. Lopez, An example in the fixed point theory of polyhedra, Bull. Amer. Math. Soc. 73 (1967), 922-924.

4. J. P. Serre, Homologic singuliere des espaces fibers, Ann. of Math. (2) 54 (1951), 425-505.

5. Shi Gen Hua, On the least number of fixed points and Nielsen numbers, Chinese Math. 8 (1966), 234-243.

Received August 18, 1969 . This paper was supported in part by the National Science Foundation under Grant NSF GR-8427 and the Wisconsin Alumni Research Foundation.

The University of Wisconsin 


\section{PACIFIC JOURNAL OF MATHEMATICS}

\section{EDITORS}

H. SAMELSON

Stanford University

Stanford, California 94305

\section{RichaRd PIERCe}

University of Washington

Seattle, Washington 98105
J. DUGUNDJI

Department of Mathematics

University of Southern California

Los Angeles, California 90007

RICHARD ARENS

University of California

Los Angeles, California 90024

\section{ASSOCIATE EDITORS}

E. F. BECKENBACH

B. H. NeUManN

F. WOLF

K. YosHIDA

\section{SUPPORTING INSTITUTIONS}

UNIVERSITY OF BRITISH COLUMBIA CALIFORNIA INSTITUTE OF TECHNOLOGY UNIVERSITY OF CALIFORNIA MONTANA STATE UNIVERSITY

UNIVERSITY OF NEVADA

NEW MEXICO STATE UNIVERSITY

OREGON STATE UNIVERSITY

UNIVERSITY OF OREGON

OSAKA UNIVERSITY

UNIVERSITY OF SOUTHERN CALIFORNIA
STANFORD UNIVERSITY

UNIVERSITY OF TOKYO

UNIVERSITY OF UTAH

WASHINGTON STATE UNIVERSITY

UNIVERSITY OF WASHINGTON

${ }^{*} \quad{ }^{*} \quad{ }^{*}$
AMERICAN MATHEMATICAL SOCIETY
CHEVRON RESEARCH CORPORATION
TRW SYSTEMS
NAVAL WEAPONS CENTER




\section{Pacific Journal of Mathematics}

\section{Vol. 33, No. $1 \quad$ March, 1970}

Mir Maswood Ali, On some extremal simplexes ................... 1

Silvio Aurora, On normed rings with monotone multiplication........... 15

Silvio Aurora, Normed fields which extend normed rings of integers....... 21

John Kelly Beem, Indefinite Minkowski spaces..................... 29

T. F. Bridgland, Trajectory integrals of set valued functions ........... 43

Robert Jay Buck, A generalized Hausdorff dimension for functions and sets ......................................... 69

Vlastimil B. Dlab, A characterization of perfect rings . . . . . . . . . . . . 79

Edward Richard Fadell, Some examples in fixed point theory ............ 89

Michael Benton Freeman, Tangential Cauchy-Riemann equations and uniform approximation ............................. 101

Barry J. Gardner, Torsion classes and pure subgroups ................ 109

Vinod B. Goyal, Bounds for the solution of a certain class of nonlinear

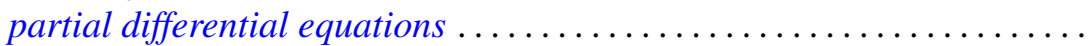

Fu Cheng Hsiang, On C, 1 summability factors of Fourier series at a given

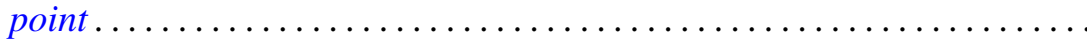

Lawrence Stanislaus Husch, Jr., Homotopy groups of PL-embedding

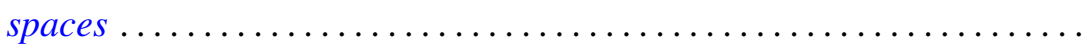

Daniel Ralph Lewis, Integration with respect to vector measures..........

Marion-Josephine Lim, $\mathscr{L}-2$ subspaces of Grassmann product spaces

Stephen J. Pierce, Orthogonal groups of positive definite multilinear functionals

W. J. Pugh and S. M. Shah, On the growth of entire functions of bounded index.

Siddani Bhaskara Rao and Ayyagari Ramachandra Rao, Existence of triconnected graphs with prescribed degrees . . .

Ralph Tyrrell Rockafellar, On the maximal monotonicity of subdifferential

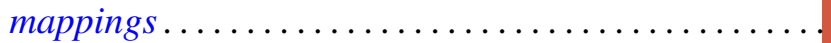

R. Shantaram, Convergence of a sequence of transformations of distribution functions. II ...............................

Julianne Souchek, Rings of analytic functions..............

Ted Joe Suffridge, The principle of subordination applied to functions of several variables...

Wei-lung Ting, On secondary characteristic classes in cobordism

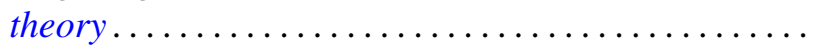

Pak-Ken Wong, Continuous complementors on $B^{*}$-algebras ...

Miyuki Yamada, On a regular semigroup in which the idempotents form a band. 